\section{Mitophagie et contrôle qualité des mitochondries}

Pierre Vigié $^{1,2}$, Nadine Camougrand ${ }^{1,2}$

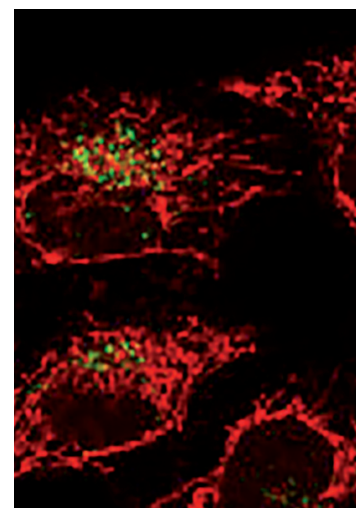

${ }^{1}$ CNRS, IBGC, UMR5095,

1, rue Camille Saint-Saëns,

F-33000 Bordeaux, France.

${ }^{2}$ Université de Bordeaux, IBGC, UMR5095,

1 , rue Camille Saint-Saëns,

F-33000 Bordeaux, France.

n.camougrand@ibgc.cnrs.fr.

tains acides aminés et lipides. En plus de son rôle vital pour la cellule, la mitochondrie est également impliquée dans la mort cellulaire programmée de type I, ou apoptose. Durant l'apoptose, la perméabilisation de la membrane externe de la mitochondrie libère le cytochrome $c$ et les facteurs apoptogéniques qui engagent le programme de mort cellulaire. Les mitochondries de mammifères sont également impliquées dans la biosynthèse des hormones stéroïdes et dans l'homéostasie du calcium. L'ensemble de ces réactions métaboliques est rendu possible grâce à de nombreux transporteurs présents dans les membranes mitochondriales, assurant les échanges entre la mitochondrie et le cytosol.

La mitochondrie est la principale source intracellulaire d'espèces activées de l'oxygène (ou ROS, reactive oxygen species). En conditions physiologiques, des ions superoxydes sont constamment générés au niveau de la chaîne respiratoire mitochondriale. Ces ROS peuvent présenter soit des effets bénéfiques, soit des effets toxiques pour la cellule. Les dommages oxydatifs et les biomolécules oxydées sont, aujourd'hui, considérés comme des contributeurs potentiels, ou des facteurs associés, à certaines pathologies comme les maladies neurodégénératives et le diabète ou encore à l'apparition de cancers. L'accumulation, au cours de la vie des cellules, de dommages liés aux ROS, est également souvent perçue comme l'une des causes majeures du vieillissement cellulaire.

\section{Le contrôle qualité des mitochondries}

Au vu de la place et du rôle des mitochondries dans l'homéostasie cellulaire, le contrôle qualité et la régulation du renouvellement de ces organites sont particulièrement importants. Le contrôle qualité des mitochondries s'effectue à différents niveaux (Figure 1). Tout d'abord, les mitochondries possèdent un ensemble de protéases et de chaperonnes localisées dans les différents compartiments mitochondriaux qui participent au bon fonctionnement mitochondrial. Parmi 


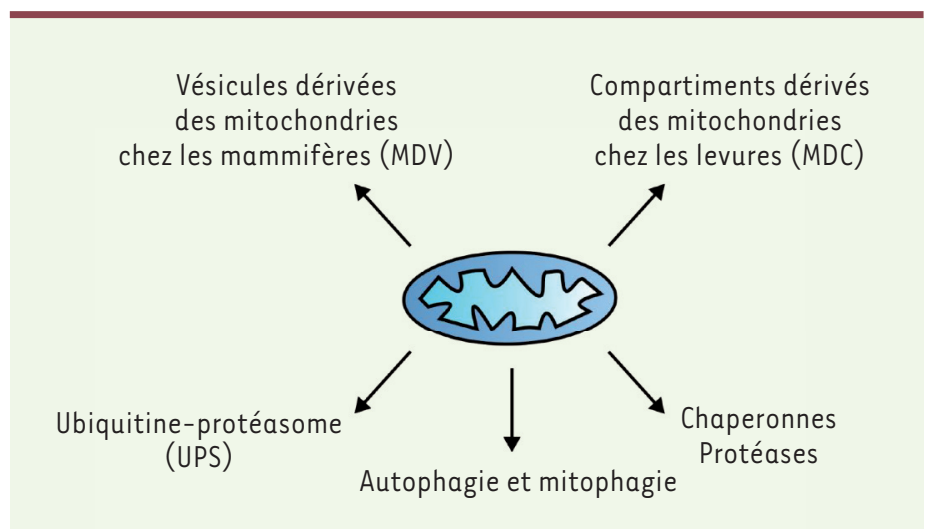

Figure 1. Le contrôle qualité des mitochondries. Le contrôle qualité des mitochondries peut s'opérer au niveau moléculaire par le biais de chaperonnes et protéases, du système ubiquitine-protéasome, mais aussi au niveau de l'organite entier par l'autophagie ou la mitophagie.

celles-ci, certaines contrôlent l'import des protéines dans la mitochondrie, d'autres jouent un rôle soit dans leur maturation, soit dans leur assemblage dans les différents complexes de la membrane interne, soit encore, dans la dégradation de peptides ou de protéines mal repliées. Le second processus qui intervient dans le contrôle qualité des mitochondries est le système ubiquitine-protéasome (ou UPS). Ce système est responsable de la dégradation de protéines à courte durée de vie, mal repliées ou endommagées qui doivent être ubiquitinylées. La fixation de l'ubiquitine sur ces protéines est orchestrée par des ubiquitine ligases ( $\varepsilon 1, \varepsilon 2$ et $\varepsilon 3)$. $\varepsilon 1$ et $\varepsilon 2$ activent l'ubiquitine par un processus qui dépend de l'ATP. $\varepsilon 3$ effectue la dernière étape en liant le groupe carboxylique de la partie C-terminale de l'ubiquitine sur l'amine de la chaîne latérale d'une lysine de la protéine qui est ciblée. Certaines ubiquitine ligases, dont MULAN ${ }^{1}$, MARCHV/MITOL et Mdm30, ont été localisées au niveau de la membrane externe de la mitochondrie. Ces ligases affectent la dynamique mitochondriale en ubiquitinylant des protéines impliquées dans les processus de fusion et de fission des mitochondries [1]. Elles assurent le contrôle qualité des protéines de la membrane externe mitochondriale. Il a également été suggéré que le système ubiquitine-protéasome régulerait d'autres protéines mitochondriales présentes dans les autres compartiments, comme l'OSCP (oligomycin sensitivity-conferring protein, une protéine matricielle), l'endonucléase $\mathrm{G}$ (une protéine de l'espace inter-membranaire), les protéines Ucp2 (uncoupling protein 2) et Ucp3 (à la membrane interne) mais le mécanisme n'a pas encore été élucidé [2]. Récemment, l'équipe du Dr Ciechanover a également montré, chez la levure Saccharomyces cerevisiae, la présence de protéines ubiquitinylées dans la matrice mitochondriale et identifié l'ubiquitine ligase Dmalp [3, 4].

\section{La mitophagie, contrôle qualité au niveau de l'organite}

Les chaperonnes, les protéases et le système ubiquitine-protéasome agissent au niveau moléculaire en surveillant les protéines. Qu'en est-

\footnotetext{
${ }^{1}$ Voir Glossaire, page 237
}

il de la surveillance au niveau de l'organite? Lorsque les dommages mitochondriaux sont trop importants, et souvent irréparables, il est nécessaire pour la cellule de mettre en place un autre processus afin d'éliminer ces mitochondries devenues défaillantes. L'autophagie est l'un des processus de dégradation cellulaire qui a été conservé chez l'ensemble des organismes eucaryotes. Elle implique une machinerie moléculaire et des compartiments de dégradation spécifiques, la vacuole chez les levures et les plantes, les lysosomes chez les mammifères. Les premiers événements d'autophagie ont été observés au milieu du xxe siècle. Ce n'est cependant que depuis le début des années 2000 que le processus est très étudié. La macroautophagie est considérée comme la principale forme d'autophagie. Elle met en jeu la formation de membranes, appelées phagophores, qui se referment en séquestrant du contenu cytoplasmique et forment des vésicules, appelées autophagosomes. Ces autophagosomes vont ensuite fusionner avec le lysosome, ou la vacuole, et leur contenu est ensuite dégradé grâce aux différentes enzymes présentes dans ces compartiments. De nombreuses maladies telles que la maladie de Parkinson, la maladie d'Alzheimer ou des cancers ont été associées à des défauts de l'autophagie $[5,6]$.

Longtemps l'autophagie a été considérée comme servant à dégrader, de manière aléatoire, des composants cytosoliques (protéines, organites, ribosomes, etc.). Cependant, de nombreuses études ont rapporté des événements de dégradation qui ciblent spécifiquement des constituants cytoplasmiques et des organites comme, par exemple, les ribosomes, le peroxysome, le noyau, le réticulum endoplasmique ou la mitochondrie [7]. Les espèces eucaryotes ont ainsi développé un processus de dégradation spécifique permettant de cibler les mitochondries, appelé mitophagie.

Les premières observations de mitochondries dégradées sélectivement par autophagie ont été réalisées chez Saccharomyces cerevisiae, en 2004, lorsque cette levure était cultivée en présence d'une source de carbone strictement respiratoire et soumise à une carence nutritive [8]. Le terme mitophagie, qui signifie dégradation sélective des mitochondries par autophagie, a, par la suite, été introduit en 2005 par John Lemasters [9]. La levure a permis de mettre en évidence un très grand nombre de protéines clés impliquées soit directement dans la machinerie de dégradation, soit dans la régulation des processus autophagiques. En 2007, Lemasters a observé, dans des hépatocytes de rat mis en condition de carence ou après un dommage ciblé provoqué par un laser, que certaines mitochondries étaient séquestrées dans des autophagosomes [10]. Depuis, le nombre de 


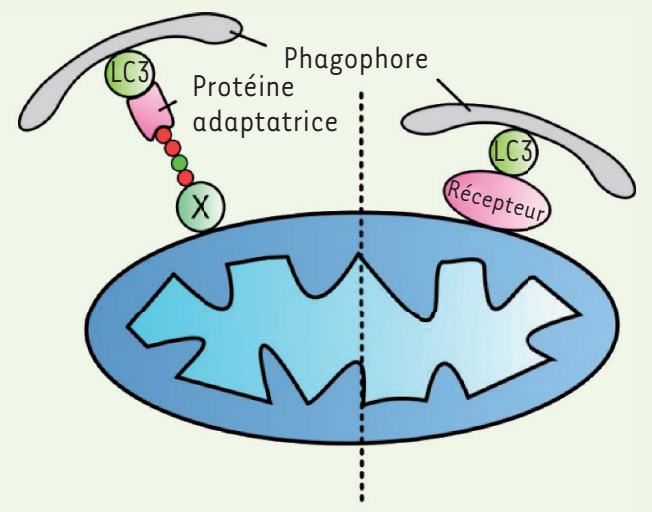

Figure 2. Les deux catégories de mitophagie dans les cellules de mammifères. La dégradation des mitochondries par mitophagie s'effectue soit par ubiquitinylation de protéines mitochondriales externes, reconnues par des protéines adaptatrices, capables d'interagir avec la protéine LC3 (microtubule-associated protein 1A/1B-light chain 3) ancrée aux membranes du phagophore, soit directement par le biais de récepteurs localisés au niveau de la membrane externe de la mitochondrie, leur interaction avec la protéine LC3 assurant le recrutement de la mitochondrie au sein du phagophore.

données relatives à ce processus n'a cessé d'augmenter, notamment en ce qui concerne la compréhension de son rôle dans le contrôle qualité des mitochondries et son lien avec de nombreuses pathologies. Pour être dégradées par mitophagie, les mitochondries doivent être étiquetées. Chez les mammifères, certaines protéines de la membrane externe mitochondriale sont, pour cela, ubiquitinylées. Elles sont alors reconnues par des protéines liant spécifiquement l'ubiquitine ou des récepteurs spécifiques de la mitophagie présents dans la membrane externe de la mitochondrie. L'interaction de ces effecteurs avec LC3 (microtubule-associated protein lA/lB-light chain 3), une protéine localisée dans les membranes des autophagosomes et qui leur est caractéristique, permettrait le recrutement et la séquestration des mitochondries (Figure 2).

Dans les cellules de mammifères, la mitophagie peut s'opérer de deux manières : la mitophagie dépendante du couple de protéines PINKI (PTEN-induced putative kinase 1)/Parkin, et la mitophagie indépendante de ce couple, régie par des récepteurs spécifiques (Figure 3).

\section{La mitophagie dépendante du couple PINK1/Parkin}

PINKI (PTEN-induced putative kinase 1 ou park 6) et PARKIN (ou park 2) sont deux gènes qui présentent des mutations dans certaines formes familiales de la maladie de Parkinson. Des travaux effectués chez la drosophile ont montré, en 2006, que PINKI et PARKIN étaient liés génétiquement et qu'ils étaient en relation avec le métabolisme mitochondrial [11-13]. En 2007, l'équipe de Youle a mis en évidence leur rôle dans la mise en œuvre de la mitophagie dans des cellules humaines en culture [14]. Au cours des années qui ont suivi, de nombreuses données ont permis d'appréhender les différentes étapes du processus. La protéine PINKI est une sérine/thréonine kinase considérée comme une protéine majeure du contrôle qualité des mitochondries. Elle est très rapidement dégradée lorsque les mitochondries sont saines. Dans ces conditions physiologiques, le renouvellement de PINKI est rapide et la protéine est peu détectable $[15,16]$. En revanche, lorsque les mitochondries subissent des dommages, comme la perte $\mathrm{du}$ potentiel membranaire mitochondrial, PINKI s'ancre au niveau de leur membrane externe et phosphoryle à la fois la protéine Parkin et l'ubiquitine. Parkin est une $\varepsilon 3$ ubiquitine ligase qui est activée par phosphorylation et possède une forte affinité pour l'ubiquitine phosphorylée en position 65 (pS65-ubiquitine). Parkin ubiquitinyle ensuite des substrats protéiques, situés au niveau de la membrane externe de la mitochondrie, et fournit I'ubiquitine, substrat de la protéine PINKI. Le signal d'induction de la mitophagie est donc amplifié. Les protéines de la membrane externe mitochondriale qui auront été ubiquitinylées seront alors soit dégradées par le protéasome, soit reconnues par des protéines ayant une affinité avec l'ubiquitine comme Optineurin (OPTN) et NDP52 (nuclear domain 10 protein 52). Cette interaction permettra ainsi d'adresser les mitochondries vers l'autophagosome afin qu'elles soient dégradées, grâce à l'interaction de OPTN et NDP52 avec la protéine $L C 3$ présente dans les membranes du phagophore (Figure 3A). Un certain nombre de protéines ajoutées au modèle par la suite, participent à la régulation du processus comme HSPAIL, BAG4, ATPIFl, SIAH3, TOMM22, TOMM7², la déubiquitine ligase USP8 et les protéases spécifiques de l'ubiquitine USP30 et USP15 [17-21]. Les conditions utilisées pour réaliser ces expériences et induire la mitophagie sont drastiques : les mitochondries sont en effet endommagées par dissipation totale du potentiel membranaire sous l'action du carbonyl cyanide m-chlorophenyl hydrazone (CCCP) et les protéines PINKI et Parkin ont souvent été surexprimées. Bien qu'elles aient permis d'élucider les bases moléculaires impliquées dans la voie de mitophagie dépendant du couple PINKl/Parkin, ces expériences ne reflètent pas les conditions physiologiques. Récemment, il a été montré qu'en endommageant les mitochondries avec un mélange constitué d'antimycine A et d'oligomycine, deux drogues qui inhibent respectivement la chaîne respiratoire et l'ATP synthase, la mitophagie pouvait être induite uniquement en présence de PINKI, la protéine kinase TBKI jouant un rôle important dans l'initiation du processus. TBKl, qui est activée lors de dommages mitochondriaux, interagirait plus particulièrement avec les protéines adaptatrices OPTN et NDP52, et serait capable de les phosphoryler afin d'augmenter leur affinité pour l'ubiquitine [22, 23].

${ }^{2}$ Voir Glossaire, page 237. 


\section{A Mitophagie dépendante de PINK1/Parkin}

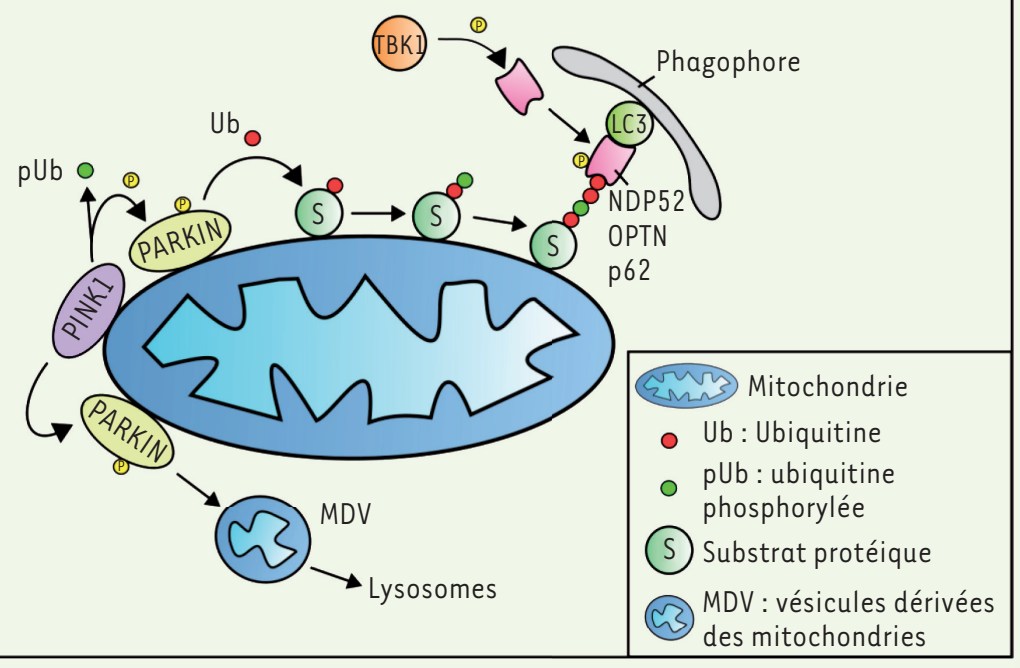

B Mitophagie indépendante de PINK1/Parkin

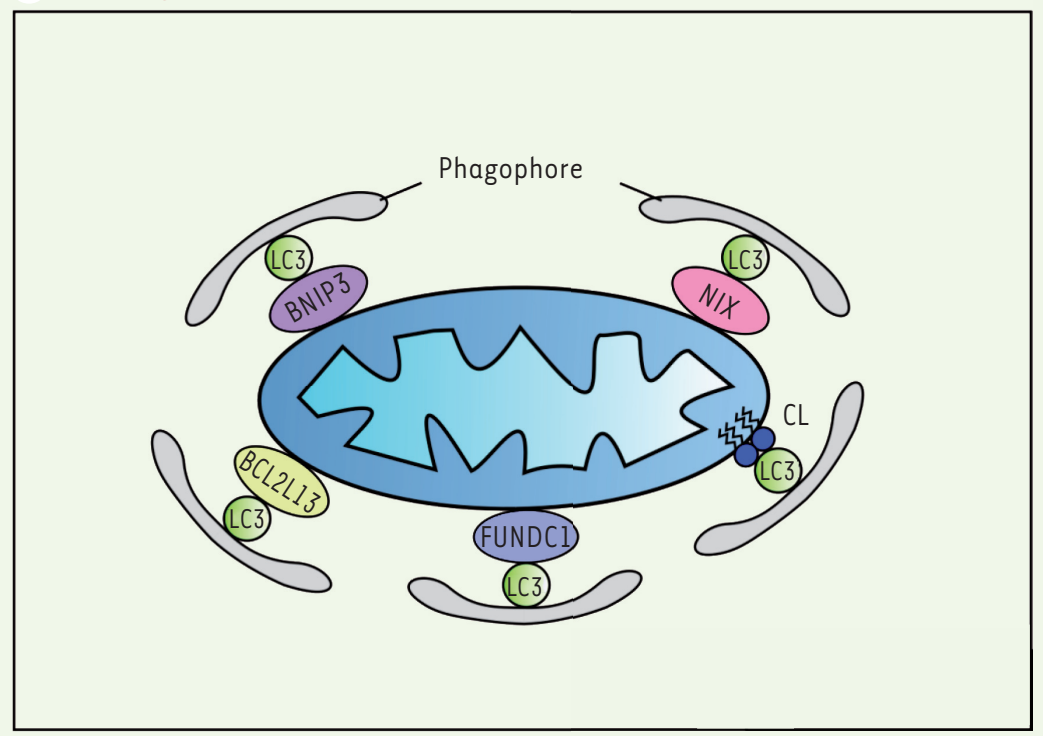

Figure 3. La mitophagie dépendante et indépendante du couple de protéines PINKI/Parkin. La mitophagie peut s'effectuer par l'intermédiaire du couple de protéines PINK1/Parkin, la protéine PINKl est recrutée au niveau de la membrane externe de la mitochondrie, phosphorylant à la fois l'ubiquitine et la protéine Parkin, activant cette dernière $(\boldsymbol{A})$. Parkin va ensuite lier l'ubiquitine aux substrats protéiques de la membrane externe, le complexe formé étant alors reconnu par les protéines adaptatrices NDP52, OPTN et p62, elles-mêmes activées par phosphorylation par TBKl. L'interaction avec la protéine LC3 ancrée dans les membranes du phagophore permet ensuite le recrutement et la séquestration des mitochondries au sein du phagophore. Le couple de protéines PINK1/Parkin n'est pas indispensable à l'induction de la mitophagie (B). En effet, la protéine LC3 serait capable d'interagir directement avec des récepteurs spécifiques localisés au niveau de la membrane externe de la mitochondrie. Ces récepteurs peuvent être protéiques (BNIP3, BCL2L13, FUNDC1 et NIX) ou lipidiques (cardiolipine). CL : cardiolipine; PINKI : PTEN (phosphatase and tensin homolog)-induced putative kinase 1 ; NDP52 : nuclear domain 10 protein 52 ; OPTN : optineurin; p62 : sequestosome-1; TBK1: TANK binding kinase 1; LC3 : microtubuleassociated protein 1A/1B-light chain 3; BNIP3: $B C L 2$ ( $B$-cell lymphoma 2)-interacting protein 3 ; BCL2L13 : BCL2 like 13; FUNDC1 : FUN14 domain containing 1 ; NIX : NIP3 (nineteen $K D$ interacting protein-3)-like protein $X$.

cellule. La fusion permet aux mitochondries endommagées de mêler leur matériel à celui de mitochondries saines et d'assurer ainsi l'homogénéité de la population mitochon-

Cette cascade PINKl/ubiquitine phosphorylée/récepteurs OPTN/NDP52 phosphorylés est suffisante pour induire la mitophagie dans ces conditions. L'addition de Parkin dans cette boucle, amplifie en fait l'ubiquitination des protéines et, par conséquent, le niveau de mitophagie.

\section{La dynamique mitochondriale et la mitophagie}

Les mitochondries sont des organites très dynamiques, en perpétuel mouvement, dont le nombre et la morphologie dépendent principalement de mécanismes de fusion et de fission dont les acteurs sont connus (Dnml, Fisl Fzol, et d'autres pour la levure; Drpl, Fisl, Opal et mitofusines pour les mammifères). L'équilibre entre fusion et fission dépend des conditions physiologiques ou de l'environnement cellulaire. Il peut également renseigner sur l'état de stress de la driale. En cas de dommages, la fission permettrait, cette fois-ci, à la mitochondrie de se diviser de façon asymétrique afin de former une mitochondrie saine, présentant un potentiel transmembranaire élevé, et une mitochondrie endommagée, avec un faible potentiel et incapable de refusionner. La fission mitochondriale serait, dans les cellules de mammifères, un pré-requis pour la mitophagie. Ainsi, Twig et ses collaborateurs ont observé que des mitochondries endommagées ne pouvant plus refusionner étaient dégradées par mitophagie, cette dégradation dépendant de la machinerie de fission [24]. De même, la fusion, permettant la formation de mitochondries tubulaires, les protège de la dégradation par autophagie lors d'une carence nutri- 
tive $[25,26]$. Des récepteurs impliqués dans la mitophagie peuvent également interagir avec les acteurs de la dynamique mitochondriale, comme Drpl ou Opal [27]. L'équipe de Kanki a cependant montré que, chez Saccharomyces cerevisiae, l'absence de la protéine de fission Dnml n'empêchait pas l'induction de la mitophagie en phase stationnaire de croissance et la retardait en condition de carence [28]. Cette équipe a également observé que, dans différents types de cellules de mammifères dépourvues de la protéine de fission Drpl, la mitophagie est induite par l'hypoxie ou par la chélation du fer [28]. Les relations entre la dynamique mitochondriale et la mitophagie semblent donc plus complexes que le modèle proposé par Twig et ses collaborateurs [24].

\section{La mitophagie indépendante du couple PINK1/Parkin : caractérisation de récepteurs spécifiques}

La mitophagie peut être déclenchée de manière indépendante du couple PINKl/Parkin. En effet, des récepteurs présents sur la membrane externe de la mitochondrie peuvent être reconnus directement par LC3, provoquant le recrutement et la séquestration des mitochondries au sein des autophagosomes (Figure 3B). Quatre protéines, localisées au niveau de la membrane externe de la mitochondrie, ont été identifiées: FUNDCl (FUN14 domain-containing 1) [29], NIX (NIP3like protein X, ou BNIP3L) [30], BNIP3 (BCL2-interacting protein 3) [31] et BCL2L13 (BCL2-like 13) [32]. Toutes ces protéines possèdent un domaine d'interaction avec la protéine $L C 3$ appelé $L I R$ domain pour LC3-interacting region. Des modifications post-traductionnelles ayant un rôle dans l'induction de la mitophagie ont été mises en évidence pour FUNDCl et BNIP3. Ces deux protéines sont, respectivement, déphosphorylées et phosphorylées lors d'une perte du potentiel mitochondrial ou d'une hypoxie, ce qui augmente leur affinité pour la protéine LC3. Chez S. cerevisiae, la protéine Atg32p, considérée comme le récepteur mitochondrial nécessaire à l'induction de la mitophagie $[33,34]$, possède également un domaine LIR et interagit avec Atg8, I'homologue de LC3 chez la levure, pour promouvoir la séquestration des mitochondries. Dans les cellules de mammifères, BCL2L13 n'a été caractérisée que très récemment. Elle serait l'homologue d'Atg32p. $\varepsilon$ effet, l'expression orthologue de BC2L13, chez la levure, permet de restaurer le défaut de mitophagie de levures dépourvues d'expression d'Atg32p (4atg32), confortant son rôle potentiel de récepteur pour la mitophagie. Comme BNIP3, NIX est impliquée à la fois dans la mort cellulaire programmée et dans la mitophagie. En tant que récepteur mitochondrial, NIX joue un rôle dans la maturation des érythrocytes en permettant l'élimination des mitochondries $[35,36]$. Très récemment, Cecconi et ses collaborateurs ont montré que la protéine pro-autophagique Ambral, dont une partie est localisée dans la membrane externe mitochondriale, interagissait avec LC3 et pouvait promouvoir la mitophagie de façon dépendante ou indépendante de la voie PINKl/ Parkin [37].

À côté des récepteurs protéiques, la cardiolipine, un phospholipide présent exclusivement dans la mitochondrie, serait impliquée dans l'induction de la mitophagie dans les cellules neuronales humaines
[38]. Des traitements par divers inducteurs de mitophagie provoquent, en effet, l'externalisation de la cardiolipine de la membrane interne vers la membrane externe de la mitochondrie. La cardiolipine pouvant interagir avec LC3, cette translocation pourrait représenter un des signaux d'induction de la mitophagie.

De nombreuses données ont donc permis de caractériser les différentes voies de mitophagie et leurs effecteurs. Les processus mis en jeu dialoguent entre eux. Ainsi, dans un modèle d'hypoxie, la protéine NIX serait requise pour l'induction de l'autophagie et la translocation de Parkin à la mitochondrie [39]. BNIP3L/NIX pourrait également être ubiquitinylée par Parkin et induire la mitophagie [40]. Ces résultats montrent le grand niveau de complexité dans la mise en place de la mitophagie.

\section{Les MDV (mitochondrial-derived vesicles) et les MDC (mitochondrial-derived compartments)}

Dans les cellules de mammifères, le contrôle qualité des mitochondries fait intervenir, en plus de la mitophagie, des vésicules dérivées des mitochondries (ou MDV pour mitochondrial-derived vesicles) [41, 42]. Les mitochondries seraient en effet capables d'envoyer des vésicules contenant du matériel mitochondrial vers les lysosomes et les peroxysomes. Si les vésicules dirigées vers les lysosomes sont destinées à être dégradées, aucun rôle précis n'a été découvert pour celles qui sont orientées vers les peroxysomes. Les MDV peuvent être générées lors d'un excès de production de ROS, induits par exemple par l'antimycine A, un inhibiteur du complexe III de la chaîne respiratoire mitochondriale. Ces vésicules permettraient d'éliminer un excès de composants mitochondriaux endommagés et constitueraient une première ligne de défense avant d'éliminer entièrement l'organite défaillant, la machinerie autophagique n'étant pas requise pour générer ces MDV. Elles transporteraient des composants provenant de la matrice, de la membrane externe et/ou de la membrane interne de la mitochondrie. Les mécanismes de formation de ces vésicules restent encore à élucider. Cependant, l'activité d'ubiquitination de la protéine Parkin serait nécessaire à leur mise en place. L'hypothèse de la formation des MDV propose que, lors d'un défaut touchant les mécanismes d'import mitochondriaux, dû à un excès d'agrégats, de protéines ou de lipides oxydés, ou à une saturation des mécanismes de protection matriciels comme ceux impliquant les chaperonnes, PINKI ne serait plus dégradée par le protéasome. La protéine resterait alors localisée au niveau de la membrane externe de la mitochondrie, ce qui induirait le recrutement de Parkin [43] qui exercerait 
son activité d'ubiquitination, ciblant des protéines de la membrane externe mitochondriale. La cardiolipine oxydée produirait de l'acide phosphatidique, un phospholipide connu pour favoriser les courbures membranaires, lui-même oxydé. L'excès d'acide phosphatidique serait à l'origine de la génération des MDV. Des études complémentaires sont cependant nécessaires pour confirmer cette hypothèse.

Les compartiments dérivés des mitochondries (ou MDC pour mitochondrial-derived compartments) ont été caractérisés très récemment chez la levure S. cerevisiae âgée. La formation des MDC qui séquestrent une catégorie de protéines bien précises a été observée lors de l'utilisation de la concanamycine A, un inhibiteur de l'ATPase vacuolaire, provoquant une augmentation $\mathrm{du} \mathrm{pH}$ du lumen vacuolaire. Même si les protéines nécessaires à la mise en place des MDC chez la levure sont différentes de celles impliquées chez les mammifères, respectivement Tom70 et PINKl/Parkin, de nombreuses similitudes sont à noter avec les MDV qui ont été caractérisées chez les mammifères [44].

\section{Perspectives}

Considérant le rôle fondamental des mitochondries, il est important de comprendre comment le contrôle qualité de ces organites se met en place, en particulier au niveau de tissus ou de cellules où la demande énergétique est élevée. De plus en plus d'évidences montrent que des aberrations dans la mise en place de ce contrôle sont associées à de nombreuses pathologies comme les maladies cardiovasculaires ou dégénératives. Au cours de ces dernières années, l'accumulation de données concernant la mitophagie a montré la complexité de ce processus. II devient donc important d'identifier et caractériser les différents mécanismes de la mitophagie afin de pouvoir mettre en place des cibles thérapeutiques pour le traitement de différentes pathologies humaines ayant pour origine le métabolisme mitochondrial. Bien que de nombreuses études aient déjà contribué à la compréhension des processus impliqués dans la mitophagie, de nombreux points restent encore à élucider : (1) Identifier les signaux à l'origine de l'induction du processus. Dans la majorité des études portant sur la voie PINKI/Parkin, le découplant CCCP (carbonyl cyanide m-chlorophenyl hydrazone) a été utilisé afin d'endommager les mitochondries, souvent de façon drastique, conduisant à leur dégradation totale. D'autres conditions ont été utilisées comme, par exemple, le mélange antimycine A-oligomycine, qui inhibent respectivement la chaîne respiratoire et l'ATP synthase, ou l'ajout de chélateur de cuivre ou de fer, ou encore l'accumulation de protéines agrégées dans la matrice mitochondriale. Aucune de ces conditions ne reflètent une quelconque situation physiologique. (2) Le second point important concerne le double rôle de l'ubiquitine qui marque soit les protéines afin qu'elles soient dégradées par le protéasome, soit les mitochondries endommagées pour induire la mitophagie, tout en sachant que, dans ces conditions, des protéines de la membrane externe de la mitochondrie peuvent également être dégradées par le protéasome. La vigilance dans l'utilisation d'outils permettant de suivre la mitophagie est donc primordiale. Il ne semble en effet pas très pertinent de suivre la dégradation des protéines de la membrane externe. En revanche, le suivi de protéines de la membrane interne, de la matrice, ou l'utilisation de la protéine mitochondriale fluorescente Keima, sensible au pH [45], pourraient être plus appropriés, de même que la mise en œuvre d'approches nouvelles.

Certaines questions restent en suspens, dont les réponses permettront de mieux comprendre la mitophagie: (1) Quelle est la place de la mitophagie dans le contrôle qualité des mitochondries et quel est le rôle de ce processus selon la spécificité cellulaire ou tissulaire ? (2) Comment définit-on une mitochondrie endommagée? (3) Comment la cellule détecte-t-elle les dommages mitochondriaux et à partir de quel niveau de dommages la mitophagie est-elle induite, sachant que des contrôles en amont, au niveau moléculaire, sont également réalisés par la cellule et les mitochondries ? Les réponses à toutes ces questions permettront de comprendre la mise en place de la mitophagie et sa régulation. Sachant que des défauts de mitophagie ont été reliés au développement de maladies neurodégénératives ou de cancers, il apparaît important de poursuivre l'étude de ce processus sur des modèles appropriés, en conditions normale ou de stress, afin d'élaborer de nouvelles stratégies thérapeutiques. $\diamond$

\section{SUMMARY}

Role of mitophagy in the mitochondrial quality control

Mitochondria are highly dynamic organelles that provide essential metabolic functions and represent the major bioenergetic hub of eukaryotic cells. Mitochondrial dysfunctions are implicated in numerous diseases. Therefore, maintenance of a healthy pool of mitochondria is required for cellular function and survival. Mitochondrial quality control is achieved through several mechanisms that act at different levels: proteases and chaperones, the Ubiquitin-ProteasomeSystem (UPS) and mitophagy. Multiple mitophagyinvolved programs operate independently or undergo crosstalk, and require modulated receptor activities at the outer membranes of mitochondria. In mammals, different mitophagy effectors have been characterized such as the receptors NIX, BNIP3, FUNDCl, BCL2L13, cardiolipin and the PINKl/Parkin pathway. Here we discuss the different molecular mechanisms of these mitophagy involved pathways. $\diamond$

\section{LIENS D'INTÉRÊT}

Les auteurs déclarent n'avoir aucun lien d'intérêt concernant les données publiées dans cet article.

\section{RÉFÉRENCES}

1. Yonashiro $R$, Ishido $S$, Kyo $S$, et al. A novel mitochondrial ubiquitin ligase plays a critical role in mitochondrial dynamics. EMBO J $2006 ; 25: 3618-26$.

2. Margineantu DH, Emerson CB, Diaz D, et al. Hsp90 inhibition decreases mitochondrial protein turnover. PLoS One 2007 ; 2 : el 066. 


\section{GLOSSAIRE}

Ambral : autophagy and beclin 1 regulator 1

ATPIF1 : ATPase inhibitory factor 1

BAG4 : Bcl-2 (B-cell lymphoma 2)-associated athanogene 4

BCL2L13 : $B C l$-2-like protein 13

BNIP3 : Bcl-2 interacting protein 3

Dnml : dynamin 1

Drpl : dynamin-1-like protein

Fis 1 : mitochondrial fission 1 protein

FUNDC1 : FUN14 domain containing 1

Fzol : mitofusin

HSPAlL : heat shock protein family A (Hsp70) member l like

LC3 ou MAPIA/B LC3 : microtubule-associated protein 1A/1B-light chain 3

MARCHV/MITOL : membrane-associated ring finger (C3HC4) 5

Mdm30 : mitochondrial distribution and morphology protein 30

MULAN : mitochondrial $\varepsilon 3$ ubiquitin protein ligase 1

NDP52 : nuclear domain 10 protein 52

NIX : NIP3 (nineteen KD interacting protein-3)-like protein X

Opal : dynamin-like $120 \mathrm{kDa}$ protein, mitochondrial

OPTN : optineurin

Parkin : enzyme de type $\varepsilon 3$ ubiquitine ligase

PINK1 : PTEN(phosphatase and tensin homolog-induced putative kinase 1)

SIAH3 : Seven in absentia homolog 3

TBK1 : TANK (TRAF family member-associated NF-kappa-B activator)

binding kinase 1

TOMM7 : translocase of outer mitochondrial membrane 7

TOMM22 : translocase of outer mitochondrial membrane 22

USP8 : ubiquitin specific peptidase 8

\section{RÉFÉRENCES}

3. Lehmann G, Ziv T, Braten 0 , et al. Ubiquitination of specific mitochondrial matrix proteins. Biochem Biophys Res Commun 2016 ; 475 : 13-8.

4. Lehmann $\mathrm{G}$, Udasin $\mathrm{RG}$, Ciechanover $\mathrm{A}$. On the linkage between the ubiquitin-proteasome system and the mitochondria. Biochem Biophys Res Commun $2016 ; 473: 80-6$.

5. Gallagher $L \varepsilon$, Williamson $L E$, Chan $\varepsilon y$. Advances in autophagy regulatory mechanisms. Cells 2016 ; 5 : pii: $\varepsilon 24$.

6. Levine B, Packer M, Codogno P. Development of autophagy inducers in clinical medicine. J Clin Invest $2015 ; 125: 14-24$

7. Stolz A, Andreas Ernst A, Dikic I. Cargo recognition and trafficking in selective autophagy. Nat Cell Biol $2014 ; 16: 495-501$.

8. Kissová I, Deffieu M, Manon S, et al. Uthlp is involved in the autophagic degradation of mitochondria. J Biol Chem 2004 ; 279 : 39068-74.

9. Lemasters JJ. Selective mitochondrial autophagy, or mitophagy, as a targeted defense against oxidative stress, mitochondrial dysfunction, and aging. Rejuvenation Res $2005 ; 8: 3-5$.

10. Kim I, Rodriguez-Enriquez S, Lemasters JJ. Selective degradation of mitochondria by mitophagy. Arch Biochem Biophys $2007 ; 462: 245-53$

11. Clark IE, Dodson MW, Jiang C, et al. Drosophila pinkl is required for mitochondrial function and interacts genetically with parkin. Nature $2006 ; 441$ : 1162-66.

12. Park J, Lee SB, Lee S, et al. Mitochondrial dysfunction in Drosophila PINKI mutants is complemented by parkin. Nature $2006 ; 441: 1157-61$.

13. Yang Y, Gehrke S, Imai Y, et al. Mitochondrial pathology and muscle and dopaminergic neuron degeneration caused by inactivation of Drosophila Pinkl is rescued by Parkin. Proc Natl Acad Sci USA $2006 ; 103: 10793-8$.

14. Narendra D, Tanaka A, Suen DF, et al. Parkin is recruited selectively to impaired mitochondria and promotes their autophagy. J Cell Biol $2008 ; 183: 795-803$.

15. Matsuda N, Sato S, Shiba K, et al. PINKl stabilized by mitochondrial depolarization recruits Parkin to damaged mitochondria and activates latent Parkin for mitophagy.J Cell Biol $2010 ; 189$ : 211-21.

16. Narendra DP, Jin SM, Tanaka A, et al. PINK1 is selectively stabilized on impaired mitochondria to activate Parkin. PLoS Biol $2010 ; 8$ : el000298.

17. Orvedahl A, Sumpter R Jr, Xiao G, et al. Image-based genome-wide siRNA screen identifies selective autophagy factors. Nature $2011 ; 480: 480-7$.

18. Lefebvre V, Du Q, Baird S, et al. Genome-wide RNAi screen identifies ATPase inhibitory factor 1 (ATPIF1) as essential for PARK2 recruitment and mitophagy. Autophagy $2013 ; 9: 1770-9$.
19. Hasson SA, Kane LA, Yamano K, et al. High-content genome-wide RNAi screens identify regulators of parkin upstream of mitophagy. Nature $2013 ; 504: 291-5$

20. Durcan TM, Tang MY, Pérusse JR, et al. USP8 regulates mitophagy by removing K6-linked ubiquitin conjugates from parkin. EMBO J $2014 ; 33: 2473-91$.

21. Bringol B, Tea JS, Phu L, et al. The mitochondrial deubiquitinase USP30 opposes parkin-mediated mitophagy. Nature $2014 ; 510: 370-5$.

22. Lazarou M, Sliter DA, Kane LA, et al. The ubiquitin kinase PINKI recruits autophagy receptors to induce mitophagy. Nature $2015 ; 524$ : 309-14.

23. Richter B, Sliter DA, Herhaus L, et al. Phosphorylation of OPTN by TBK1 enhances its binding to $\mathrm{Ub}$ chains and promotes selective autophagy of damaged mitochondria. Proc Natl Acad Sci USA 2016 ; 113 : 4039-44.

24. Twig $G$, Elorza A, Molina AJ, et al. Fission and selective fusion govern mitochondrial segregation and elimination by autophagy. EMBO J 2008 ; 27 : 433-46.

25. Gomes LC, Di Benedetto $G$ and Scorrano L During autophagy mitochondria elongate, are spared from degradation and sustain cell viability. Nat Cell Biol $2011 ; 13$ : 589-98.

26. Rambold AS, Kostelecky B, Elia N and Lippincott-Schwartz J Tubular networkformation protects mitochondria from autophagosomal degradation during nutrient starvation. Proc Natl Acad Sci USA 2011 ; 108 : 10190-5.

27. Chen $M$, Chen ZH, Wang yy et al. Mitophagy receptor FUNDCl regulates mitochondrial dynamics and mitophagy. Autophagy 2016; 12 : 1-14.

28. Yamashita SI, Jin X, Furukawa K, et al. Mitochondrial division occurs concurrently with autophagosome formation but independently of Drpl during mitophagy. J Cell Biol $2016 ; 215: 649-55$.

29. Liu L, Feng D, Chen G, et al. Mitochondrial outer-membrane protein FUNDC1 mediates hypoxia-induced mitophagy in mammalian cells. Nat Cell Biol $2012 ; 14: 177-85$.

30. Novak I, Kirkin V, McEwan DG, et al. Nix is a selective autophagy receptor for mitochondrial clearance. EMBO Rep 2010 ; 11 : 45-51.

31. Zhu Y, Massen S, Terenzio M, et al. Modulation of serines 17 and 24 in the LC3-interacting region of Bnip3 determines pro-survival mitophagy versus apoptosis. J Biol Chem $2013 ; 288$ : 1099-113.

32. Murakawa T, Yamaguchi 0 , Hashimoto $\mathrm{A}$, et al. $\mathrm{Bcl}-2$-like protein 13 is a mammalian Atg32 homologue that mediates mitophagy and mitochondrial fragmentation. Nat Commun $2015 ; 6$ : 7527-41.

33. Kanki T, Wang K, Cao Y, et al. Atg32 is a mitochondrial protein that confers selectivity during mitophagy. Dev Cell $2009 ; 17$ : 98-109.

34. Okamoto K, Kondo-Okamoto N, Ohsumi Y. Mitochondria-anchored receptor Atg32 mediates degradation of mitochondria via selective autophagy. Dev Cell $2009 ; 17: 87-97$.

35. Schweers RL, Zhang J, Randall MS, et al. NIX is required for programmed mitochondrial clearance during reticulocyte maturation. Proc Natl Acad Sci USA $2007 ; 104: 19500-5$

36. Sandoval H, Thiagarajan P, Dasgupta SK, et al. Essential role for NIX in autophagic maturation of erythroid cells. Nature $2008 ; 454: 232-5$.

37. Strappazzon F, Nazio F, Corrado M, et al. AMBRAl is able to induce mitophagy via LC3 binding, regardless of PARKIN and p62/SPSTM1. Cell Death Diff 2015; $22: 419-32$.

38. Chu CT, Ji J, Dagda RK, et al. Cardiolipin externalization to the outer mitochondrial membrane acts as an elimination signal for mitophagy in neuronal cells. Nat Cell Biol $2013 ; 15$ : 1197-205.

39. Ding WX, Ni HM, Li M, et al. Two distinct phases of mitophagy, reactive oxygen species-mediated autophagy induction and Parkin-Ubiquitin-p62mediated mitochondrial priming. J Biol Chem $2010 ; 285$ : 27879-90.

40. Gao F, Chen D, Si J, et al. The mitochondrial protein BNIP3L is the substrate of PARK2 and mediates mitophagy in PINK1/PARK2 pathway. Hum Mol Genet $2015 ; 24: 2528-38$.

41. Soubannier V, McLelland GL, Zunino R, et al. A vesicular transport pathway shuttles cargo from mitochondria to lysosomes. Curr Biol $2012 ; 22$ : 135-41.

42. Soubannier V, Rippstein P, Kaufman BA, et al. Reconstitution of mitochondrial derived vesicle formation demonstrates selective enrichment of oxidized cargo. PLoS One $2012 ; 7$ : e52830.

43. Sugiura A, McLelland GL, Fon $E A$, et al. A new pathway for mitochondrial quality control: mitochondrial-derived vesicle. $\varepsilon M B O J$ J $2014 ; 33$ : 2142-56.

44. Hughes AL, Hughes $C \varepsilon$, Henderson KA, et al. Selective sorting and destruction of mitochondrial proteins in aged yeast. Elife 2016 ; 5 : pii: el3943.

45. Katayama H, Kogure $\mathrm{T}$, Mizushima $\mathrm{N}$, et al. A sensitive and quantitative technique for detecting autophagic events based on lysosomal delivery. Chem Biol 2011 ; 18 : 1042-52. 